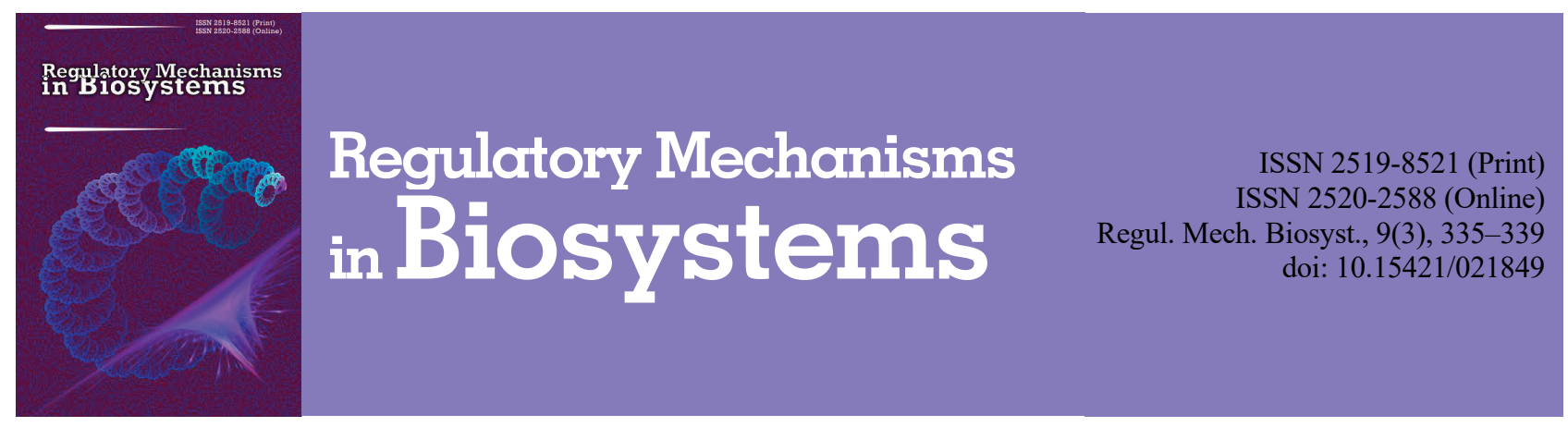

\title{
Methods for increasing the accuracy of recording the parameters of the cardiovascular system in double-beam photoplethysmography
}

\author{
Y. M. Snizhko, O. O. Boiko, N. P. Botsva, D. V. Chernetchenko, M. M. Milyh \\ Oles Honchar Dnipro National University, Dnipro, Ukraine
}

Article info

Received 12.06.2018

Received in revised form 20.07.2018

Accepted 24.07.2018

Oles Honchar Dnipro National University, Gagarin ave., 72, Dnipro, 49010, Ukraine.

Tel.: +38-056-373-12-63. E-mail:nbotsva@gmail.com

\begin{abstract}
Snizhko, Y. M., Boiko, O. O., Botsva, N. P., Chernetchenko, D. V., \& Milyh, M. M. (2018). Methods for increasing the accuracy of recording the parameters of the cardiovascular system in double-beam photoplethysmography. Regulatory Mechanisms in Biosystems, 9(3), 335-339. doi:10.15421/021849

Photoplethysmography has recently become more widespread among non-invasive methods for obtaining information on the state of physiological systems of the human body. Serial photoplethysmographs are intended for use in clinics and require special care, therefore, interest in portable media developed on the basis of modern sensors and microcontrollers is growing, which would not only make this method available for individual use, but also expand its capabilities through the use of light of various spectral ranges. Such devices require modified signal processing techniques that allow them to be used in mobile applications. The aim of the work is to develop methods for processing signals from a modern two-beam sensor operating in the red and infrared ranges for the analysis of photoplethysmography on a mobile device (smartphone or tablet). A device using the microcontroller and radio module in the Bluetooth standard allows you to continuously record pulse waves, determine the level of oxygen in the blood, calculate peak-peak intervals and heart rate. The use of the two-beam sensor for registration and the implementation of the developed signal processing methods in the Android operation system application increase the accuracy of setting the maximums on pulse curve and provide a relative error in determining the heart rate and pulse-to-pulse intervals relative to the certified electrocardiograph at $9.2 \%$ and $9.6 \%$ respectively, with an average level of interference and an average activity. An Android operation system mobile device (tablet, smartphone) allows you to visualize the measurement results, store data in the internal memory, and transfer them to the server for further processing.
\end{abstract}

Keywords: photoplethysmography; absorption spectra; pulse oximetry; photoplethysmography analysis; oxygenation; continuous registration of oxygen saturation in peripheral vessels.

\section{Introduction}

Photoplethysmography is a method for recording the optical density of tissue using a photoelectric plethysmograph. It is used to study the individual properties of regional blood circulation, the spectral properties of blood flowing through the investigated area of the body. Recently, single-beam and two-beam photoplethysmography have been widely used to determine a number of parameters of the cardiovascular system, in particular, the frequency of breathing, the heart rate, the duration of pulse-to-pulse intervals. The method of pulse oximetry, which is used in birefringence photoplethysmography, also determines the level of oxygen in the blood.

The assessment of respiratory rate, which is an important indicator for ambulatory care, can be done in real time with a signal from a portable one-beam pulse oximeter (Karlen et al., 2011; Lin et al., 2017). In this case, the definition of respiration frequency according to photoplethysmography is more reliable and has a lower error than the electrocardiography signal.

As a contactless method for determining the heart rate, photoplethysmography with a camera, is used for recording the instantaneous pulsations in the change in face skin colour during the cardiovascular wave, invisible to the eye, but measured during video recording (Aarts et al., 2013; Alghoul et al., 2017).

The variability of heart rhythm is widely used for early diagnosis of cardiovascular diseases and the evaluation of the functions of the autonomic nervous system (Bulvestre et al., 2013; Cygankiewicz et al., 2015; Goldkorn et al., 2015). The predictive value of heart rate variability has been demonstrated by numerous studies in recent years (Al-Zaiti et al., 2014; O'Neal et al., 2016; Botswa et al., 2017). The signal from which both the time domain and the frequency domain of heart rate variability are measured requires an accurate determination of the interval between consecutive cardiac contractions (Blood et al., 2015; Ha et al., 2015). The classical method involves analysing the numerical R-R intervals for electrocardiogram signals which are obtained in a laboratory (Task Force, 1996; ChuDuc et al., 2013). However, certain methodological problems associated with the recording and analysis of electrocardiograms create a barrier to the collection of large amounts of data, which are necessary for a statistically reliable comparison of the status of various subjects.

Some authors have recently preferred an alternative method for analysing variability of heart rhythm - instead of R-R intervals in the electrocardiogram, they are using time intervals between peak values in the photoplethysmography signal, which also reflects the heart rate, since the mechanical activity of the heart is related to its electrical activity (Schäfer \& Vagedes, 2013; Kavsaoğlu et al., 2016). In this case, in place of an electrocardiogram a portable photoplethysmograph is used, which operates on an optical pulse oximeter based on a smartphone or similar portable device and can serve as the basis for a new tool for screening heart rate variability indices in a nonclinical environment (Dehkordi et al., 2016; Orphanidou, 2017). A similar system typically has a photosensor on the finger for measuring the intensity of reflection, after which the signal is selected, filtered, processed and sent via a wireless channel for further processing. The system is often used on the wrist in the cuffs to make it wearable and easy to wear (Sudin et al., 2015). 
The analysis shows a high level of agreement between the peak-peak intervals obtained from an electrocardiogram and photoplethysmography (Weinschenk et al., 2016), that's why the changes in pulse rate which are determined by the photoplethysmography can be used instead of variability of heart rhythm, for a more complete evaluation of the autonomic nervous system's functions. Numerous studies suggest that photoplethysmography using pulse oximetery as a simple noninvasive method for determining the interval between successive heart contractions and the calculation of heart rate variability is more practical and reliable not only in ideal conditions for healthy subjects (Lu et al., 2009; Karlen et al., 2011; Chen et al., 2015), but also for elderly patients (Chuang et al., 2015), in non-stationary conditions (Gil et al., 2010) and in conditions of motion (Sudin et al., 2015). Such a system allows for an approximation sufficient for analyses of the temporal and frequency regions of the heart rate variability, which creates conditions for studying of heart rate variability by the photoplethysmography signal for a large number of subjects in cardiological and psychophysiological studies (Heathers, 2013), e.g. in the interpretation of emotions (Alghoul et al., 2017; Costa et al., 2018) and for the care of certain groups of patients with electrocardiogram's artefacts.

However, during the analysis it should be borne in mind that the changes in the photoplethysmography signal have some delay in time with respect to the electrocardiogram signal, which is needed for passing the wave (Selvaraj et al., 2008). In addition, physical activity and some psychological stressors decrease correlation level between the values of heart rate for photoplethysmography and heart rate variability (Schäfer \& Vagedes, 2013) as a result of changes during stressful loadings of heart rate variability parameters (Lin et al., 2014). Moreover, the effect of the breathing process also has an influence. Recently, for eliminating the influence the frequency of breathing on photoplethysmography, a new method has been proposed for patients wearing sensors (Orphanidou, 2017), but for determination of more accurate values of R-R intervals it is recommended to use the registration of photoplethysmography and electrocardiogram at the same time.

Some researchers pay attention to the fact that the heart rate variability analysis by photoplethysmography signal which is taken from wearable sensors makes the influence of noise and trends more powerful, which is why for achieving more reliable results of photoplethysmography analysis, decreasing of effect of this artefact and creating more accurate determination methods are very important tasks (Alian \& Shelley, 2014). This adds to the relevance of research into finding hardware and software methods for making more accurate recording of the photoplethysmography signal and for improving the obtaining of necessary parameters for subsequent processing.

Recently a reliable algorithm was created for fast online evaluation of the quality of the photoplethysmographic signals, which has the advantage of being used in mobile devices with low technical power (Pflugradt et al., 2015). For removing the influence of artefacts of physical motion on the results of analysis of photoplethysmography from wearable devices, a suggestion was made for recording at the same time the signal from an accelerometer with following filtration and processing methods of spectral analysis (Han \& Kim, 2012; Islam et al., 2017). It has been established that the trends of photoplethysmographic signals which occur in the low frequency range are changed during the spectral analysis, that's why they must be removed using methods of filtrations in the primary processing before starting analysis of heart rate variability (Akdemir et al., 2013). For assessing respiratory rate using the method of single-beam photoplethysmography, the algorithm which is based on Wavelet transform is very appropriate.

Among the software for the processing of signals of photoplethysmography, also it should be noted that new algorithms for the allocation of peaks in the photoplethysmography signal are based on the median method (Firoozabadi et al., 2017) and the adaptive segmentation method (Kavsaoğlu et al., 2016). The latter, according to the authors, allows you to correctly determine peaks from photoplethysmographic signals at rest even without pre-processing.

Thus, in the meantime, the interest of researchers in the registration and processing of photoplethysmography with wearable sensors based on simple mobile devices is increasing. Available hardware-software and processing algorithms allow one to perform the initial signal processing and improve its quality at the stage of preparation for the analysis of single-beam photoplethysmography. However, in the literature there is not enough information on the algorithms for improving the quality of the processing of the results of two-beam photoplethysmography. The aim of the research presented in this article is the development of methods for processing signals from a modern twobeam sensor, which work in red and infrared ranges for the analysis of photoplethysmography on a mobile device (smartphone or tablet).

\section{Materials and methods}

For registration of parameters of the cardiovascular system by the method of two-beam photoplethysmography, a device was created which contains two light-emitting diodes in a sensor, the first one is red, the second one is infrared (Fig. 1). The light-emitting diodes alternately emit pulse wavelengths of $680 \mathrm{~nm}$ (RED) and $950 \mathrm{~nm}$ (IR). After reflection from biological tissues which contain blood vessels, these pulses are recorded by a photodetector, converted into a digital signal and sent to a microcontroller. The microcontroller manages the sensor, performs the initial processing of the received signal and sends it for further processing to a mobile device which uses the operation system of Android. Transmission of processing results to a phone or tablet is made by wireless Bluetooth at a distance of up to $5 \mathrm{~m}$. The main processing of signals is carried out on the microcontroller, visualization and storage of results on a mobile device. This allows one to collect a lot of data from many experiments for further analysis. The developed device allows one to register the signal continuously for two hours.

The photoplethysmography is analysed using two algorithms: the first one can determine the level of oxygen saturation in peripheral vessels $\left(\mathrm{SpO}_{2}\right)$, the second one is the time value of systolic peaks which is needed for calculation of pulse-to-pulse intervals and heart rate.

The algorithm for determining the level of oxygen in the blood is based on the dependence of the absorption coefficient of light by different states of hemoglobin from the light-emitting diode wavelength: at a wavelength of $660 \mathrm{~nm}$ (RED), hemoglobin is absorbed about 10 times more than oxyhemoglobin and at a wavelength of $940 \mathrm{~nm}$ (IR) the absorption of oxyhemoglobin is more than hemoglobin (Fig. 2).

For non-invasive determination of the level of oxygen saturation in human blood, the sensor is placed next to the portion of the tissue which contains arterial vessels. Under these conditions, the level of the signal from the sensor is proportional to the absorption of light in the tissues and due to the presence of arterial blood circulation has two component's the constant $\left(\mathrm{A}_{\mathrm{DC}}\right)$ and the variable $\left(\mathrm{A}_{\mathrm{AC}}\right)$ (Fig. 3).

Signal valuation is used to increase the accuracy of the determination of saturation by pulse oximetry. The normalized signal $\mathrm{S}$ for each of the two wavelengths is defined as the ratio of the variable component of the corresponding $\mathrm{A}_{\mathrm{AC}}$ signal to the constant component of the $\mathrm{A}_{\mathrm{DC}}$, which are measured at the time of diastole,

$$
\mathrm{S}=\mathrm{A}_{\mathrm{AC}} / \mathrm{A}_{\mathrm{DC}} \text {. }
$$

Normed signals $S_{R E D}$ and $S_{I R}$ do not depend on the intensity of light from light-emitting diodes, but are determined only by optical properties of living tissue (Fig. 4). By their values, the ratio $\mathrm{R}$ is calculated

$$
\mathrm{R}=\mathrm{S}_{\mathrm{RED}} / \mathrm{S}_{\mathrm{IR}} \text {. }
$$

The value of $\mathrm{R}$ is related to the definition of $\mathrm{SpO}_{2}$ saturation by empirical dependence

$$
\mathrm{SpO}_{2}=\mathrm{a}-\mathrm{b} \cdot \mathrm{R},
$$

where $\mathrm{a}, \mathrm{b}$ are coefficients determined empirically during calibration depending on the certificated device (Kalakutskij \& Manelis, 1999). In this case, following the calibration of the Heaco CMS50C "Pulse Oximeter" certified device, the following coefficients are set: $\mathrm{a}=109.0$, $\mathrm{b}=11.3$. The value of $\mathrm{R}$ varies from 0.8 for $100 \%$ saturation and 9.6 for $0 \%$ saturation; $85 \%$ saturation corresponds to the value of $\mathrm{R}=2.12$.

The algorithm for detecting the peaks of systolic elevation on photoplethysmography is based on the principle of state machine, which is to monitor the state of the current signal value in time. This machine follows the loop of states, which allows us to separate parts of the photoplethysmography signal in real-time mode into five parts and research each of them individually (Fig. 5). 


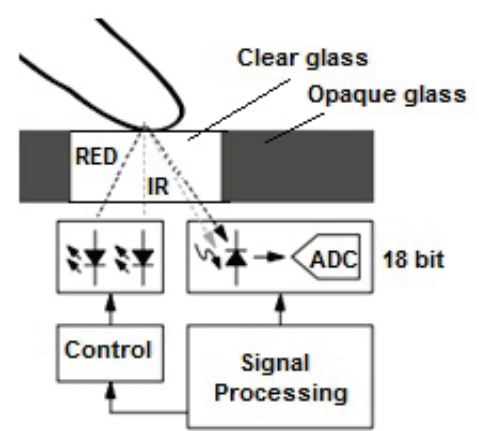

Fig. 1. Scheme of registration of photoplethysmography based on the sensor that works on the principle of reflection of light from tissues

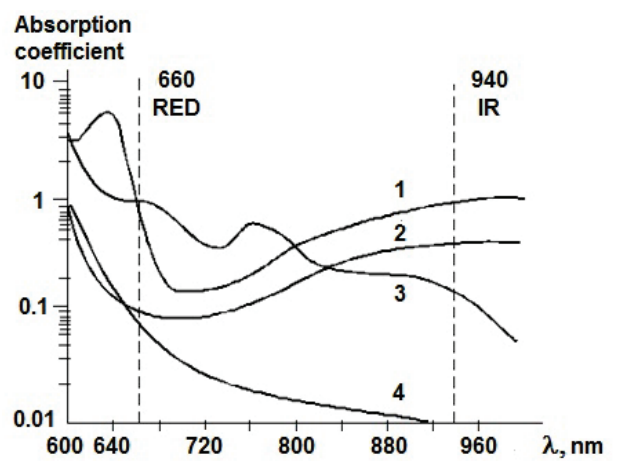

Fig. 2. Dependence of the absorption coefficient of light in different forms hemoglobin on the wavelength $\lambda$ of radiation:

1 - methemoglobin, 2 - oxyhemoglobin, 3 - hemoglobin, 4 - carboxyhemoglobin

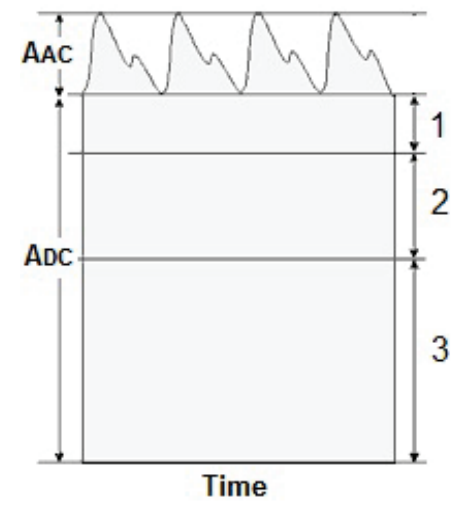

Fig. 3. Components of the signal from the sensor of the photoplethysmography: $A_{A C}$ is a variable component, $A_{D C}$ is a constant component: 1 - arterial blood, 2 - venous blood, 3 - tissue

The first state - STATE_INIT - is responsible for initializing and indicates the start of the algorithm.

The second state - STATE_WAITING - is responsible for adapting all required boundaries of the passage and seeing whether the transition to the next state has begun.

The third state - STATE_FOLLOWING_SLOPE - corresponds to the signal region where the systolic rise occurs and the transition to the next state, where the top of the systolic lift may be detected.

The fourth state - STATE MAYBE DETECTED - checks if the vertex of the systolic lift is actually recorded or the signal is still in state STATE_FOLLOWING_SLOPE. If the systolic peak is fixed, its time coordinate is stored in memory, and the algorithm goes to the next state.

The fifth state - STATE_MASKING - masks (holds) the algorithm's work for a while, after which it is re-executed.

The algorithms for determining the saturation level and the detection of peaks of photoplethysmography are developed and implemented in the Java programming language for the Android operating system.
For further analysis of the cardiovascular system, according to the time coordinates of the systolic peaks, the programmed R-R intervals and heart rate are determined.

The table shows the general characteristics of a mobile device for the registration of two-beam photoplethysmography.

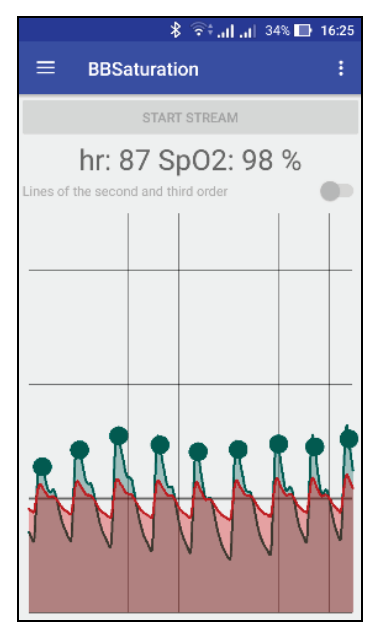

Fig. 4. View of the photoplethysmography on a mobile device: the lower signal wave corresponds to red (RED) range, and upper - to infrared (IR); the values of heart rate and saturation are calculated at the top

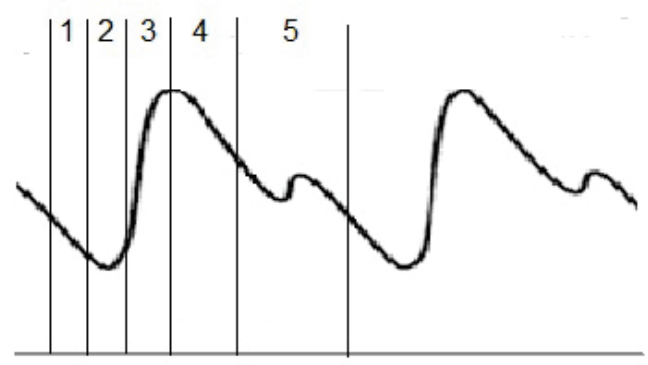

Fig. 5. The states of the algorithm for detecting the peaks of systolic lift on photoplethysmography

Table

General characteristics of the mobile device for photoplethysmography measurement

\begin{tabular}{lc}
\hline \multicolumn{1}{c}{ Parameter } & Value \\
\hline Sampling frequency, $\mathrm{Hz}$ & 50 \\
Duration of one measurement, min & $2-3$ \\
Power supply, V & $3.3<\mathrm{U}<5.0$ \\
Battery capacity, $\mathrm{mA} / \mathrm{h}$ & 160 \\
Dimensions, $\mathrm{mm}$ & $60 \times 35 \times 10$ \\
\hline
\end{tabular}

\section{Results}

During the preliminary testing of the algorithm for detecting the peaks of systolic lift and determining the heart rate on a mobile device, along with the results similar to those shown in Figure 4, it was possible to observe cases of registration of peaks that looked false (Fig. 6).

The developed algorithm can be sufficiently precise and flexible in use. For checking and analysis of the developed systolic peak detection algorithm we performed research which determined peak-peak intervals and heart rate with mobile devices for photoplethysmography and compared this with results of R-R intervals which was determined by the certified electrocardiograph CONTEC8000GW ECG Workstation.

For the experiment we took nine volunteers aged from 20 to 40 without any disorders of the cardiovascular system. For each participant about 2-3 measurements were carried out with duration 2-3 minutes using both devices for registration of the appropriate intervals and heart rate. According to the results of each measurement, pairs of mean values of intervals and heart rate were determined (Fig. 7, 8). 


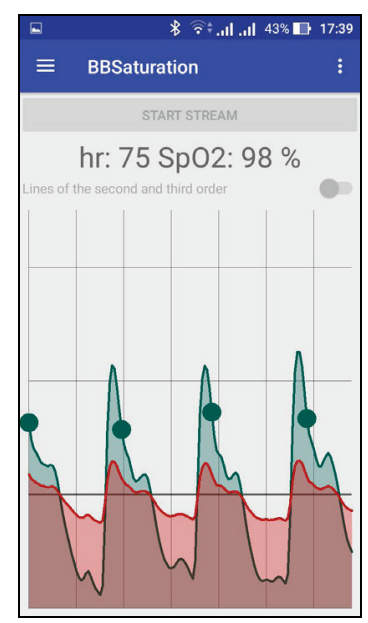

Fig. 6. Photoplethysmography with expectation interval relative to peak peak, where green dot is a peak of systolic lift

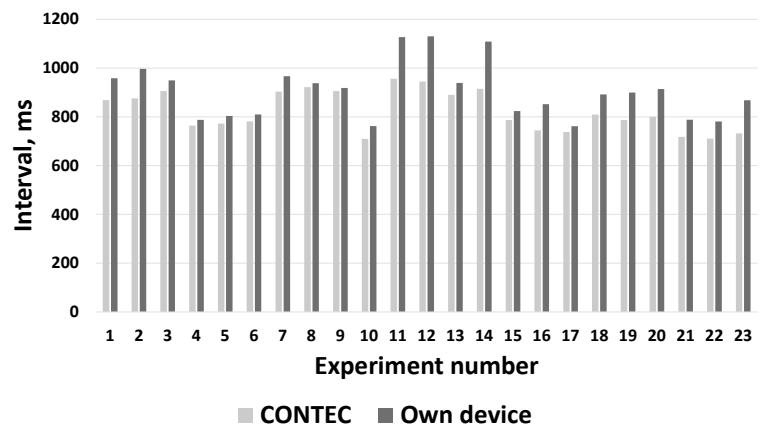

Fig. 7. Results of measurement of R-R intervals (light colour) and peak-peak intervals (dark colour) with two devices

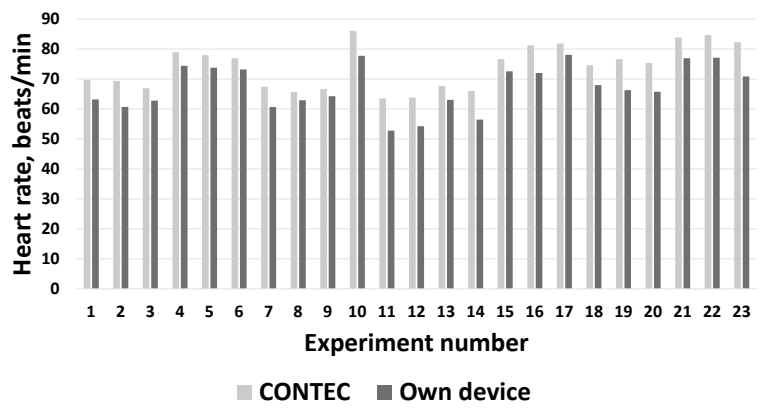

Fig. 8. Results of measurement of heart rate by two devices

Calculated from experimental data, the mean absolute and relative errors are respectively: for peak-peak intervals $79.7 \mathrm{~ms}$ and $9.6 \%$, for heart rate 9.6 beats $/ \mathrm{min}$ and $9.2 \%$

\section{Discussion}

However, it should be noted that precisely in the state where the peak of systolic lifting is detected, the algorithm provides protection against signal noise at the peaks of the wave. That is why in state STATE_MAYBE_DETECTED there is a so-called waiting interval, belonging to the current time coordinate and verified by the algorithm. If the conditions are true, this point is recorded as the peak of the systolic wave. Such a check is a significant advantage of the developed algorithm, which precisely increases its accuracy and allows one to perform the functions of determining peaks at any quality of the input signal of the photoplethysmography.

During the testing, certain weaknesses of the algorithm were also found. Firstly, the value of the waiting interval should be set depending on the quality of the output signal from the wearer. Secondly, the STATE MASKING state duration value also needs an initial definition and is significantly dependent on the peak-peak intervals. However, this disadvantage is not critical, since such a duration is sufficient to set $1 / 4$ $1 / 6$ of the average statistical value of the peak-peak interval. The obtainned results confirm the conformity of the proposed methods of processing the signal of the photoplethysmography and the performance of the device as a whole. It is seen that the developed device does not have many differences in the determination of absolute values of parameters relative to the certified electrocardiograph, and it can be noted that all deviations are unidirectional. The relative error value is almost the same as the results of comparative studies comparing electrocardiography and photoplethysmography with a relative error of $10.3 \%$ (Orphanidou, 2017).

The existing discrepancies between the results for photoplethysmography and electrocardiogram may be due to the fact that comparisons of measurements were from instruments which are used for different objects and methods of research: the cardiograph records the electrical signals of the heart, and the photoplethysmograph analyses the cardiovascular system as a whole, whose state, in particular, depends on the state of vessels. Therefore, the results can be considered acceptable.

In general, the obtained results are in agreement with the results of other authors, which determine the possibility of substituting heart rate variability analysis for electrocardiogram signals by analysis of pulse variability according to the results of photoplethysmography, but at the same time emphasize the fact that the differences between these indicators can be caused by respiration, in particular respiratory delusions and rhythmic breathing (Chen et al., 2015). There are also some differences in the spectral values of heart rate variability and pulse wave changes, mainly in the area of high respiratory rates (Gil et al., 2010). Therefore, it is obvious that the influence of breathing on the accuracy of determining the parameters of the cardiovascular system for photoplethysmography requires further research.

Studies have shown that although the photoplethysmographic signals provides accurate data for analysing the parameters of heart rate variability in ideal conditions, it is less reliable due to its vulnerability to artefacts of movements (Lu \& Yang, 2009). Along with this, it is believed that the pulse wave can be used as an electrocardiogram substitute for the analysis of heart rate variability in non-stationary conditions (Gil et al., 2010).

The development of tools for modelling realistic photoplethysmographic signals for the development and testing of photoplethysmograph-specific arrhythmia detectors can also be considered promising, taking into account the lack of annotated public databases of photoplethysmograph with arrhythmias (Soloshenko et al., 2017).

\section{Conclusion}

We developed methods of analysis of photoplethysmographic signals with modes for determination of the level of oxygination and the setting of heart rate parameters on a mobile unit that contains a twobeam sensor with red and infrared light-emitting diodes, a microcontroller and a radio module in the Bluetooth standard. The device allows you to continuously record pulse waves, determine the level of oxygen saturation, peak-peak intervals and heart rate. The use of two-beam sensors for registration and implementation of developed signal processing methods in the Android operation system application allow one not only to calculate the saturation of blood but also to increase the accuracy of setting the maximum of the corresponding pulse curves and to provide a relative error in determining the heart rate and peak-peak intervals relative to a certified electrocardiograph at $9.2 \%$ and $9.6 \%$ with an average level of interference and average muscle activity. An Android operation system mobile device (tablet, smartphone) allows one to visualize the measurement results, store data in the internal memory, and transfer them to the server for further processing.

It is possible to use the device to determine other parameters associated with different physiological processes, such as breathing rate.

\section{References}

Aarts, L. A. M., Jeanne, V., Cleary, J. P., Lieber, C., Nelson, J. S., Bambang Oetomo, S., \& Verkruysse, W. (2013). Non-contact heart rate monitorring 
utilizing camera photoplethysmography in the neonatal intensive care unit A pilot study. Early Human Development, 89(12), 943-948.

Akdemir Akar, S., Kara, S., Latifoğlu, F., \& Bilgiç, V. (2013). Spectral analysis of photoplethysmographic signals: The importance of preprocessing. Biomedical Signal Processing and Control, 8(1), 16-22.

Alghoul, K., Alharthi, S., Al Osman, H., \& El Saddik, A. (2017). Heart rate variability extraction from videos signals: ICA vs. EVM Comparison. IEEE Access, 5, 4711-4719.

Alian, A. A., \& Shelley, K. H. (2014). Photoplethysmography. Best Practice and Research Clinical Anaesthesiology, 28(4), 395-406.

Al-Zaiti, S. S., Fllavollita, J. A., Canty, J. J. M., \& Carey, M. G. (2014). Electrocardiographic predictors of sudden and non-sudden cardiac death in patients with ischemic cardiomyopathy. Heart and Lung: The Journal of Acute and Critical Care, 43(6), 527-533.

Blood, J. D., Wu, J., Chaplin, T. M., Hommer, R., Vazquez, L., Rutherford, H. J. V., Mayes, L. C., \& Crowley, M. J. (2015). The variable heart: High frequency and very low frequency correlates of depressive symptoms in children and adolescents. Journal of Affective Disorders, 186, 119-126.

Botsva, N., Naishtetik, I., Khimion, L., \& Chernetchenko, D. (2017). Predictors of aging based on the analysis of heart rate variability. Pacing and Clinical Electrophysiology, 40(11), 1269-1278.

Bulvestre, M., Leprêtre, P. M., \& Ghannem, M. (2013). 243: Heart rate variability analysis could help to determine the ventilatory threshold in patients with heart failure. Archives of Cardiovascular Diseases Supplements, 5(1), 82.

Chen, X., Huang, Y. Y., Yun, F., Chen, T. J., \& Li, J. (2015). Effect of changes in sympathovagal balance on the accuracy of heart rate variability obtained from photoplethysmography. Experimental and Therapeutic Medicine, 10(6), 23112318

Chuang, C. C., Ye, J. J., Lin, W. C., Lee, K. T., \& Tai, Y. T. (2015). Photoplethysmography variability as an alternative approach to obtain heart rate variability information in chronic pain patient. Journal of Clinical Monitoring and Computing, 29(6), 801-806.

ChuDuc, H., NguyenPhan, K., \& NguyenViet, D. (2013). A review of heart rate variability and its applications. APCBEE Procedia, 7, 80-85.

Costa, A., Rincon, J. A., Carrascosa, C., Julian, V., \& Novais, P. (2018). Emotions detection on an ambient intelligent system using wearable devices. Future Generation Computer Systems. In press.

Cygankiewicz, I., Corino, V., Vazquez, R., Bayes-Genis, A., Mainardi, L., Zareba, W. de Luna, A. B., \& Platonov, P. G. (2015). Reduced irregularity of ventricular response during atrial fibrillation and long-term outcome in patients with heart failure. The American Journal of Cardiology, 116(7), 1071-1075.

Dehkordi, P., Garde, A., Karlen, W., Petersen, C. L., Wensley, D., Dumont, G. A. \& Mark Ansermino, J. (2016). Evaluation of cardiac modulation in children in response to apnea/hypopnea using the Phone Oximeter TM. Physiological Measurement, 37(2), 187-202.

Firoozabadi, R., Helfenbein, E. D., \& Babaeizadeh, S. (2017). Efficient noisetolerant estimation of heart rate variability using single-channel photoplethysmography. Journal of Electrocardiology, 50(6), 841-846.

Gil, E., Orini, M., Bailón, R., Vergara, J. M., Mainardi, L., \& Laguna, P. (2010). Photoplethysmography pulse rate variability as a surrogate measurement of heart rate variability during non-stationary conditions. Physiological Measurement, 31(9), 1271-1290.

Goldkorn, R., Naimushin, A., Shlomo, N., Dan, A., Oieru, D., Moalem, I., Rozen, E., Gur, I., Levitan, J., Rosenmann, D., Mogilewsky, Y., Klempfner, R., \& Goldenberg, I. (2015). Comparison of the usefulness of heart rate variability versus exercise stress testing for the detection of myocardial ischemia in patients without known coronary Artery Disease. The American Journal of Cardiology, 115(11), 1518-1522.

Ha, J. H., Park, S., Yoon, D., \& Kim, B. (2015). Short-term heart rate variability in older patients with newly diagnosed depression. Psychiatry Research, 226, $484-488$.

Han, H., \& Kim, J. (2012). Artifacts in wearable photoplethysmographs during daily life motions and their reduction with least mean square based active noise cancellation method. Computers in Biology and Medicine, 42(4), 387-393.
Heathers, J. A. J. (2013). Smartphone-enabled pulse rate variability: An alternative methodology for the collection of heart rate variability in psychophysiological research. International Journal of Psychophysiology, 89(3), 297-304.

Islam, M. T., Zabir, I., Ahamed, S. T., Yasar, M. T., Shahnaz, C., \& Fattah, S. A. (2017). A time-frequency domain approach of heart rate estimation from photoplethysmographic (PPG) signal. Biomedical Signal Processing and Control, 36, 146-154

Kalakutskij, L. I., \& Manelis, J. S. (1999). Apparatura i metody klinicheskogo monitoringa [Apparatus and methods of clinical monitoring]. Samara State Aerospace University, Samara (in Russian)

Kavsaoğlu, A. R., Polat, K., \& Bozkurt, M. R. (2016). An innovative peak detection algorithm for photoplethysmography signals: An adaptive segmentation method. Turkish Journal of Electrical Engineering and Computer Sciences, 24(3), 1792-1796.

Lin, F., Heffner, K., Mapstone, M., Chen, D.-G. (Din), \& Porsteisson, A. (2014). Frequency of mentally stimulating activities modifies the relationship between cardiovascular reactivity and executive function in old age. The American Journal of Geriatric Psychiatry, 22(11), 1210-1221.

Lin, Y.-D., Chien, Y.-H., \& Chen, Y.-S. (2017). Wavelet-based embedded algorithm for respiratory rate estimation from PPG signal. Biomedical Signal Processing and Control, 36, 138-145.

Lu, G., \& Yang, F. (2009). Limitations of oximetry to measure heart rate variability measures. Cardiovascular Engineering, 9(3), 119-125.

Lu, G., Yang, F., Taylor, J. A., \& Stein, J. F. (2009). A comparison of photoplethysmography and ECG recording to analyse heart rate variability in healthy subjects. Journal of Medical Engineering and Technology, 33(8), 634-641.

O’Neal, W. T., Chen, L. Y., Nazarian, S., \& Soliman, E. Z. (2016). Reference ranges for short-term heart rate variability measures in individuals free of cardiovascular disease: The multi-ethnic study of atherosclerosis (MESA) Journal of Electrocardiology, 49(5), 686-690.

Orphanidou, C. (2017). Derivation of respiration rate from ambulatory ECG and PPG using ensemble empirical mode decomposition: Comparison and fusion. Computers in Biology and Medicine, 81, 45-54.

Pflugradt, M., Moeller, B , \& Orglmeister, R. (2015). OPRA: A fast on-line signa quality estimator for pulsatile signals. IFAC-PapersOnLine, 48(20), 459-464.

Schäfer, A., \& Vagedes, J. (2013). How accurate is pulse rate variability as an estimate of heart rate variability?: A review on studies comparing photoplethysmographic technology with an electrocardiogram. International Journal of Cardiology, 166(1), 15-29.

Selvaraj, N., Jaryal, A., Santhosh, J., Deepak, K. K., \& Anand, S. (2008). Assessment of heart rate variability derived from finger-tip photoplethysmography as compared to electrocardiography. Journal of Medical Engineering and Technology, 32(6), 479-484.

Sološenko, A., Petrénas, A., Marozas, V., \& Sörnmo, L. (2017). Modeling of the photoplethysmogram during atrial fibrillation. Computers in Biology and Medicine, 81, 130-138

Sudin, S., Aziz, F., Mohd Hishamuddin, N. A., Ahmad Saad, F. S., Md Shakaff, A. Y., Zakaria, A., \& Salleh, A. F. (2015). Wearable heart rate monitor using photoplethysmography for motion. In: IECBES 2014, Conference Proceedings - 2014 IEEE Conference on Biomedical Engineering and Sciences. Pp. 1015-1018.

Task force of the European Society of Cardiology and the North American Society of Pacing and Electrophysiology. Heart rate variability. Standards of measurement, physiological interpretation and clinical use (1996). Circulation, 93 1043-1065.

Verma, A., Cabrera, S., Mayorga, A., \& Nazeran, H. (2013). A robust algorithm for derivation of heart rate variability spectra from ECG and PPG signals. In: Proceedings - 29th Southern Biomedical Engineering Conference, SBEC 2013. Pp. 35-36

Weinschenk, S. W., Beise, R. D., \& Lorenz, J. (2016). Heart rate variability (HRV) in deep breathing tests and 5-min short-term recordings: agreement of ear photoplethysmography with ECG measurements, in 343 subjects. European Journal of Applied Physiology, 116(8), 1527-1535. 